\title{
Therapeutic nuclear medicine expands to breast cancer
}

\author{
Marc André Mahé • Albert Lisbona • \\ Jean-François Chatal
}

Published online: 5 December 2009

(C) Springer-Verlag 2009

After breast-conserving surgery the standard treatment, for the majority of patients with early breast cancer, is adjuvant whole-breast radiotherapy delivering a dose of about $50 \mathrm{~Gy}$ and followed by an additional local boost dose of 10-20 Gy applied to the tumour bed. Such adjuvant treatment allows reduction in the absolute risk of local recurrence by $19 \%$ in patients with negative lymph nodes and $33 \%$ in patients with positive lymph nodes. Moreover, the gain in survival has been shown to be $5.4 \%$ at 15 years [1].

This treatment irradiates both the clusters of malignant cells located inside or in close proximity to the tumour bed and remote sites in the breast. Moreover, it can be completed with irradiation of lymph node areas according to the anatomical localization of the tumour and histoprognostic factors such as axillary lymph node involvement.

The limitations of this whole-breast radiotherapy include delay exceeding 8 weeks in initiating treatment, which may decrease the probability of local control [2], and overall treatment time, which is a problem for patients continuing their professional activity, for elderly patients and for patients living far from the radiotherapy centre. Moreover, cosmetic

This Editorial Commentary refers to the article http://dx.doi.org/ $10.1007 / \mathrm{s} 00259-009-1260-4$

\section{A. Mahé}

Department of Radiotherapy, René Gauducheau Cancer Center, Nantes-Saint-Herblain, France

\section{A. Lisbona}

Medical Physics Department, René Gauducheau Cancer Center, Nantes-Saint-Herblain, France

\section{J.-F. Chatal $(\bowtie)$}

GIP Arronax,

1, rue Aronnax, BP 10112,

44817 Nantes-Saint- Herblain, France

e-mail: chatal@arronax-nantes.fr outcomes are not always excellent due to fibrosis, and finally transportation costs may have a significant financial impact.

Over the past few years accelerated partial-breast irradiation has been used as an alternative to whole-breast radiotherapy aiming at shortening overall treatment time and reducing the workload of radiotherapy departments and cost. Several techniques have been implemented and are being used for specific indications [3].

The interstitial multicatheter technique has the longest reported experience exceeding 5 years. It is an invasive procedure requiring good clinical experience and consists in inserting some plastic catheters loaded with ${ }^{192} \mathrm{Ir}$ into the tumour bed. This technique is flexible, which allows adapting the conformation of the irradiated volume to the anatomical characteristics and having a reproducible geometry of the dose distribution.

Intraoperative radiotherapy using electrons produced in a linear accelerator or $50 \mathrm{kV} \mathrm{X-rays} \mathrm{is} \mathrm{another} \mathrm{technique}$ (Intrabeam ${ }^{\circledR}$ ) with a rationale based on the immediate irradiation of the tumour bed after surgical resection of the tumour. The intraoperative condition allows delivering a high dose to a limited volume and sparing the surrounding normal tissue.

Another technique uses a balloon catheter (called MammoSite $\left.{ }^{\circledR}\right)$ filled with ${ }^{192}$ Ir and inserted into the resection cavity. This is a simple method requiring a short learning curve and with a well-standardized dosimetry but which may not be adapted to specific individual target volumes. Moreover, this technique may lead to unfavourable cosmetic outcomes.

Finally, 3-D conformal external beam radiotherapy and intensity modulated radiation therapy (IMRT) are noninvasive techniques allowing homogeneous irradiation of the tumour bed volume and good cosmetic outcome.

In summary, if we consider all of these invasive and noninvasive accelerated partial-breast irradiation techniques, 
their main advantages, with regard to conventional wholebreast irradiation, include a shortened overall treatment time and a decreased irradiation of apparently normal breast and adjacent organs. Their main disadvantage is the possibility of having occult foci of cancer cells in the part of the breast outside of the tumour bed and which should not been treated. That is why, before reaching sufficiently long follow-up times that allow a definite opinion to be reached about the long-term efficacy and side effects of accelerated partial-breast irradiation, it has been recommended that its use be restricted to a well-defined subgroup of low-risk patients. For that purpose two consensus statements have been recently published [3, 4]. Patients who could be considered for a technique of accelerated partial-breast irradiation should be committed to a long-term follow-up to evaluate the recurrence rate and toxicity.

In this issue, the paper by Giovanni Paganelli et al. [5] reports on an innovative irradiation technique which is an attractive compromise between the commonly used wholebreast radiotherapy and the aforementioned accelerated partial-breast irradiation techniques. This new technique is based on a pretargeting method [6] using the very highaffinity streptavidin-biotin binding which had been initiated by the same group in the early 1990s [7]. The first application was three-step radioimmunotherapy using a biotinylated antibody molecule, avidin/streptavidin and ${ }^{90}$ Y-biotin. For this new clinical application of pretargeting in breast irradiation, avidin is injected first into and around the tumour bed and ${ }^{90}$ Y-biotin is injected intravenously 1 day later and diffuses rapidly to the breast tissue where it binds to the "pretargeted" avidin. This results, like for intraoperative techniques, in an immediate irradiation avoiding the risk of local recurrence which has been debated with delayed radiotherapy.

Combining the pretargeted technique with a shortened external beam radiotherapy over 4 weeks, the authors calculated a total biological effective dose of $70 \mathrm{~Gy}$, similar to the dose delivered by conventional external beam radiotherapy extending over 7 weeks. Such extrapolation should be considered cautiously due to radiobiological differences between internal and external radiotherapy.
On the whole, this intraoperative avidination for radionuclide therapy (IART) technique allows shortening the treatment time from 7 to 4 weeks with regard to the conventional external beam radiotherapy, which can be of real interest for patients who are working or have family obligations, for capacity of radiotherapy institutions and for treatment costs. However, it will take some time before such a new approach is approved for routine use. As claimed by the authors of the paper, a randomized trial including many patients and extending over a long follow-up period will be necessary to validate routine application.

Finally, this study illustrates the expansion of radionuclide therapy and prefigures its integration into a multimodality approach with external beam radiotherapy and chemotherapy.

\section{References}

1. Clarke M, Collins R, Darby S, Davies C, Elphinstone P, Evans $\mathrm{E}$, et al. Effects of radiotherapy and of differences in the extent of surgery for early breast cancer on local recurrence and 15-year survival: an overview of the randomised trials. Lancet 2005;366:2087-106.

2. Huang J, Barbera L, Brouwers M, Browman G, Mackillop WJ. Does delay in starting treatment affect the outcomes of radiotherapy? A systematic review. J Clin Oncol 2003;21:555-63.

3. Sauer R, Sautter-Bihl ML, Budach W, Feyer P, Harms W, Souchan $\mathrm{R}$, et al. Accelerated partial breast irradiation: consensus statement of 3 German Oncology societies. Cancer 2007;110:1187-94.

4. Smith BD, Arthur DW, Buchholz TA, Haffty BG, Hahn CA, Hardenbergh $\mathrm{PH}$, et al. Accelerated partial breast irradiation consensus statement from the American Society for Radiation Oncology (ASTRO). J Am Coll Surg 2009;209:269-77.

5. Paganelli G, De Cicco C, Ferrari ME, Carbone G, Pagani G, Leonardi $\mathrm{MC}$, et al. Intraoperative avidination for radionuclide treatment as radiotherapy boost in breast cancer: results of a phase II study with (90)Y-labeled biotin. Eur J Nucl Med Mol Imaging 2009.

6. Goldenberg DM, Sharkey RM, Paganelli G, Barbet J, Chatal JF. Antibody pretargeting advances cancer radioimmunodetection and radioimmunotherapy. J Clin Oncol 2006;24:823-34.

7. Paganelli G, Magnani P, Zito F, Villa E, Sudati F, Lopalco L, et al. Three-step monoclonal antibody tumor targeting in carcinoembryonic antigen-positive patients. Cancer Res 1991;51:5960-6. 